\title{
CHARACTERS AND GALOIS INVARIANTS OF REGULAR DESSINS
}

\author{
Manfred STREIT and Jürgen WOLFART
}

\begin{abstract}
We describe a new invariant for the action of the absolute Galois groups on the set of Grothendieck dessins. It uses the fact that the automorphism groups of regular dessins are isomorphic to automorphism groups of the corresponding Riemann surfaces and define linear representations on the space of holomorphic differentials. The characters of these representations give more precise information about the action of the Galois group than all previously known invariants, as it is shown by a series of examples. These examples have their own interest because the Galois action on them is described only using properties of the fixed points in the canonical model, without the explicit knowledge of equations.
\end{abstract}

\section{Regular dessins, Galois actions, and charac- ters}

Let $Y$ be a compact Riemann surface or equivalently a smooth projective algebraic curve defined over a subfield of $\mathbb{C}$. We call it a Belyi surface if a Belyi function $\beta: Y \rightarrow \mathbb{P}^{1}$ exists on $Y$, i.e. non-constant, meromorphic and ramified at most above $0,1, \infty$. Equivalent definitions (see e.g. [JS] or [Wo1]) are

1. as an algebraic curve, $Y$ can be defined over a number field

2. as a Riemann surface, $Y$ is isomorphic to a quotient $\Gamma \backslash \mathcal{H}$ of the upper half plane $\mathcal{H}$ by a subgroup $\Gamma$ of finite index in a Fuchsian triangle group $\triangle \subset P S L_{2} \mathbb{R}$

3. the conformal structure of $Y$ is uniquely determined by a (Grothendieck) dessin, a bipartite graph $D$, that is with white

1991 Mathematics Subject Classification: 14H30, 20H10, 30F10, 11F70, 11F80.

Servicio de Publicaciones. Universidad Complutense. Madrid, 2000 
and black vertices - every white vertex has only black neighbours and vice versa - and $Y-D$ a finite disjoint union of simply connected cells.

In fact, every Belyi function defines a dessin given by the inverse image $\beta^{-1}[0,1]$ of the real interval $[0,1]$. The white and black vertices are the points of $\beta^{-1}\{0\}$ and $\beta^{-1}\{1\}$ respectively, and any dessin on a compact orientable 2 -manifold determines uniquely a conformal structure and a Belyi function on it with that property.

One of the main problems in the theory of Grothendieck's dessins d'enfants is to understand the action of the absolute Galois group $\mathrm{Gal} \overline{\mathbb{Q}} / \mathbb{Q}$ on them. In fact, every $\sigma \in \mathrm{Gal} \overline{\mathbb{Q}} / \mathbb{Q}$ maps every Belyi surface $Y$ onto another Belyi surface $Y^{\sigma}$ via the action on the points of the nonsingular projective algebraic curves and on the coefficients of their defining equations, and the action on the coefficients of the Belyi function $\beta$ under consideration gives a new Belyi function $\beta^{\sigma}$ on $Y^{\sigma}$, hence defining a Galois conjugate dessin $D^{\sigma}$. We think that a description of this Galois action should be decomposed into the action on Riemann surfaces with many automorphisms or regular dessins, see below, and the action on their quotients.

To that aim we recall first that the property that the Riemann surface $X$ of genus $g>1$ has many automorphisms may be defined in several equivalent ways [Wo1].

1. Every proper local deformation $X_{\epsilon}$ of $X$ (corresponding to a point $P\left(X_{\epsilon}\right)$ in a small punctured neighbourhood of $P(X)$ in the moduli space $\mathcal{M}_{g}$ ) has an automorphism group which is strictly smaller than Aut $X$.

2. The universal covering group $N \subset P S L_{2}(\mathbb{R})$ of $X$ is a normal subgroup of a Fuchsian triangle group $\Delta$ (and Aut $X \cong \Delta / N=: G$ if $\Delta$ is the normalizer of $N$ in $P S L_{2} \mathbb{R}$, in particular if $\Delta$ is a maximal triangle group).

3. $X \rightarrow$ Aut $X \backslash X$ is a Belyi covering, i.e. there exists a Belyi function $B: X \rightarrow \mathbb{P}^{1}$ defining a normal covering, given by the canonical projection $N \backslash \mathcal{H} \rightarrow \Delta \backslash \mathcal{H}=G \backslash X$ where we identify the target quotient space with $\mathbb{P}^{1}$ and the fixed point orbits of $\Delta$ with $0,1, \infty$ respectively. 
4. The conformal structure on $X$ is characterized by a regular dessin what means that the dessin has an automorphism group acting transitively on the edges.

5. At least for genus $g>3$ the corresponding point $P(X)$ of the moduli space is an isolated singularity of $\mathcal{M}_{g}$ in the sense of Zariski [Po] (not to be confused with the more restricted notion of topologically isolated points of the set of singular points in $\mathcal{M}_{g}$ as considered by Kulkarni $[\mathrm{K}]$ ).

In the present paper, the most important characterizations are those given in 2 . and 3 . We remark in passing that $\Delta$ is uniquely determined by its signature $\langle p, q, r\rangle$ up to conjugation in $P S L_{2} \mathbb{R}$ whence we will simply write $\Delta=\langle p, q, r\rangle$. Since $N$ is torsion free, $G=$ $\Delta / N$ has three generators $g, h, k$ with $g h k=1$ and of orders $p, q, r$ respectively. These orders are of course also the ramification orders of $B$ above $0,1, \infty$, respectively.

Every dessin has a regular dessin as a finite cover. Equivalently, every Belyi surface $Y$ is covered by a Riemann surface with many automorphisms $X$ taking the normalization of the covering given by the original Belyi function $\beta: Y \rightarrow \mathbb{P}^{1}$. The resulting normal covering map $B: X \rightarrow \mathbb{P}^{1}$ is again a Belyi function whose dessin $B^{-1}[0,1]$ gives the regular covering of the original dessin $\beta^{-1}[0,1]$. Its automorphism group $G$, i.e. the covering group of $B$, is isomorphic to its hypermap group ( = monodromy group of $B$ ) and also to the hypermap group of the original dessin $\beta^{-1}[0,1]$, namely the monodromy group of $\beta$, and can also be identified with a subgroup of the automorphism group of the Belyi surface $X$, for details see Theorem 2 of [Wo2]. Now Galois conjugations of $Y$ and of the Belyi function $\beta$ induce Galois conjugations of $X$, and if $X$ has many automorphisms then so has $X^{\sigma}$ for any Galois conjugation $\sigma$, the automorphism group $G$ of $X$ being $\sigma$-conjugate to an automorphism group $G^{\sigma}$ of $X^{\sigma}$. The canonical Belyi function

$B: X \rightarrow G \backslash X \cong \mathbb{P}^{1}$ is conjugate to $B^{\sigma}: X^{\sigma} \rightarrow G^{\sigma} \backslash X^{\sigma} \cong \mathbb{P}^{1}$

which is again a canonical Belyi function, with the same ramification orders as $B$ (canonical means that $B$ is uniquely determined by $X$ and $G$ which is Aut $X$ in most cases - uniquely determined up to automorphisms of $\mathbb{P}^{1}$ possibly permuting $0,1, \infty$, compare Remark 1 at the end 
of Section 3). Thus $\sigma$ induces an obvious mapping of regular dessins $D$ to regular dessins $D^{\sigma}$ with isomorphic automorphism groups $G \cong G^{\sigma}$ and preserving the orders of the generators of $G$ or equivalently, the valencies of $D$. Therefore, the problem of understanding these Galois actions can be divided into two parts.

- Consider isomorphic Belyi surfaces as equivalent and describe the action of $\mathrm{Gal} \overline{\mathbb{Q}} / \mathbb{Q}$ on the equivalence classes of Belyi surfaces with many automorphisms $X$. In particular, determine the subgroup $H$ of all Galois conjugations $\sigma$ sending $X$ to an isomorphic Belyi surface $X^{\sigma}$, and the fixed field $M(X)$ of $H$, the moduli field of $X$. By a result essentially due to Coombes and Harbater ([DE], [Wo1]) a Belyi surface with many automorphisms $X$ has a model defined over its moduli field.

- Describe the action of $H$ on the intermediate coverings of $B: X \rightarrow$ $\mathbb{P}^{1}$, i.e. on all quotients of $X$ by subgroups of $G$. Equivalently, describe the action of $H$ on the subgroups of the automorphism group $G$ of $X$ (clearly, the automorphisms of $X$ - defined over $\overline{\mathbb{Q}}$ - form a group invariant under conjugation by $H$ ).

In this paper the first problem will be considered for a special family of Riemann surfaces with a given automorphism group. The second problem is connected to the theory of $G$-coverings and will not be considered here (in the examples treated below this action will be rather trivial). For regular dessins on Riemann surfaces with many automorphisms, the isomorphism class of the automorphism group $G$, together with the order of their generators is obviously invariant under Galois conjugation - for an account of the actually known invariants of dessins under the action of $\mathrm{Gal} \overline{\mathbb{Q}} / \mathbb{Q}$ see [JSt]. Unfortunately these known invariants do not form a complete list of Galois invariants in general. They are sufficient to characterize Galois orbits in some important special cases such as elliptic curves with complex multiplication and uniform dessins (Singerman/Syddall [SSy]) or Hurwitz curves with automorphism groups $P S L_{2} \mathbb{F}_{q}$ over a finite field $\mathbb{F}_{q}$ (Streit [St2]). They are also trivially sufficient for curves $X$ with many automorphisms in small genera $g, 1<g<5$, since $X$ is uniquely determined by its automorphism group Aut $X$ at least in these cases. (Then, by the way, 
$M(X)=\mathbb{Q}$, so $X$ may be defined over the rationals). As far as we know, historically the first examples where these invariants were known to be insufficient are 'Leila's flowers', see p.71 of [Sps].

One aim of our investigation is the careful study of an infinite family of Riemann surfaces with automorphism groups

$$
G \cong Z_{p} \rtimes Z_{q}
$$

which are the semi-direct products of the multiplicative cyclic groups of prime orders $q$ and $p \equiv 1 \bmod q$. We give a very explicit description of these surfaces and we try to collect as much information about these surfaces as one can possibly extract. Partly this is done to exhibit the power of a new Galois invariant we propose using the representations

$$
\psi_{k}: G \rightarrow G L\left(H^{0}\left(X, \Omega^{k}\right)\right)
$$

on the vector space of holomorphic differentials of degree $k \in \mathbb{N}$ defined by

$$
\psi_{k}(g): \omega \mapsto \omega \circ g^{-1}
$$

for all $g \in G$. For a given group $G$ we obtain a sequence of representations $\left(\psi_{k}\right)_{k \in \mathbb{N}}$ and call two such sequences $\left(\psi_{k}\right)_{k \in \mathbb{N}},\left(\widetilde{\psi_{k}}\right)_{k \in \mathbb{N}}$ equivalent iff there is a $\tau \in$ Aut $G$ such that $\left(\psi_{k}\right)_{k \in \mathbb{N}} \cong\left(\widetilde{\psi_{k}} \circ \tau\right)_{k \in \mathbb{N}}$, i.e. iff we can find a fixed $\tau$, such that $\psi_{k}$ is isomorphic to $\widetilde{\psi_{k}} \circ \tau$ for all $k \in \mathbb{N}$. This implies $\left(t r \psi_{k}\right)_{k \in \mathbb{N}}=\left(t r \widetilde{\psi_{k}} \circ \tau\right)_{k \in \mathbb{N}}$ and we call these two sequences equivalent as well. The composition of the representations $\psi_{k}$ with a fixed $\tau \in$ Aut $G$ corresponds to the existence of different homomorphisms of $\Delta$ onto $G$ with the same kernel. Here only outer automorphisms are relevant as for all inner automorphisms we have $\psi_{k} \cong \psi_{k} \circ \tau$ and $\operatorname{tr} \psi_{k}(g)=\operatorname{tr} \psi_{k}(\tau(g))$ for all $g \in G$. With respect to our second aim we want to formulate our

Main Observation. Let $X, X^{\prime}$ be Riemann surfaces of genus $g>1$ with many automorphisms and

$$
G=\Delta / N \cong \Delta / N^{\prime}
$$

for a Fuchsian triangle group $\Delta$ containing the universal covering groups $N, N^{\prime}$ of $X$ and $X^{\prime}$ as normal subgroups. Let $\psi_{k}, \psi_{k}^{\prime}$ be the representations of $G$ on the spaces of holomorphic k-differentials on $X, X^{\prime}$ respectively. If

$$
X^{\prime} \cong X^{\sigma} \text { for some } \sigma \in \mathrm{Gal} \overline{\mathbb{Q}} / \mathbb{Q},
$$


then there exists an $\tau \in$ Aut $G$ such that the characters of $\psi_{k}, \psi_{k}^{\prime}$ satisfy

$$
\operatorname{tr} \psi_{k}^{\prime}(g)=\sigma\left(\operatorname{tr} \psi_{k}(\tau(g))\right) \text { for all } g \in G \text { and all } k,
$$

i.e. $\left(\psi_{k}^{\sigma}\right)_{k \in \mathbb{N}},\left(\psi_{k}^{\prime}\right)_{k \in \mathbb{N}}$, respectively $\left(\operatorname{tr} \psi_{k}^{\sigma}\right)_{k \in \mathbb{N}},\left(\operatorname{tr} \psi_{k}^{\prime}\right)_{k \in \mathbb{N}}$ are equivalent.

The proof is almost obvious using the action of $\sigma$ on the curve $X$, on a basis of its differentials which may be assumed to be defined over $\overline{\mathbb{Q}}$, on the elements $g$ of the automorphism group $G$, giving a coefficientwise action of $\sigma$ on the representation matrices $\psi_{k}(g)$. Much less obvious is the question if the Galois orbit of this character is a useful invariant, i.e. if characters can distinguish Galois orbits whose other invariants are the same, and if they can be explicitly calculated. The latter question of how to calculate this invariant will be discussed in some detail in Section 6. All informations about this invariant are encoded in the behaviour of elements of the cartographic group $G$ by operating on the cosets of the cyclic groups generated by the standard generators of $G$. We will classify all regular dessins and Riemann surfaces having the described groups as automorphism groups (Section 2). This family shows that characters are in fact a powerful tool, but the dessins are interesting in its own right by various reasons.

- The action of $\mathrm{Gal} \overline{\mathbb{Q}} / \mathbb{Q}$ on the curves of the family can be described without explicit knowledge of the defining equations. The method is based on ideas of the first author concerning canonical models, fixed points and multipliers, first applied in [St2] on a series of Hurwitz curves. Another possible access could be the use of Belyı's cyclotomic character.

- Consequently, the moduli field can be determined explicitly. As explained above, it coincides with the minimal field of definition (Theorems 1 and 3).

- This field of definition is never $\mathbb{Q}$, so Hilbert's irreducibility theorem fails to produce the groups $G$ as Galois groups over $\mathbb{Q}$ by specializing extensions of function fields ramified over three points only.

- With Eichler's trace formula, the character of $\psi$ can be calculated and decomposed into irreducible components (Theorem 5). 
- In general, there is no hope that the Galois orbits of the characters in the Main Observation form a complete system of Galois invariants for dessins. But in the case of our family of examples they correspond in fact bijectively to the Galois orbits of the dessins in question, already for 1-differentials (Theorem 4), although both the number of Galois orbits and their length grow linearly with $q$.

- On the other hand, with methods explained in [JSt] it is easy to prove that the previously known best Galois invariants for dessins - the cartographic groups introduced in [JSt] - are the wreath product $(G \times G) \rtimes S_{2}$ for almost all Galois orbits treated in Theorem 3 (i.e. if the indices of $X_{n, t, v}$ are pairwise distinct). So they are unable to distinguish different Galois orbits in most of our cases.

- On the way, we will meet some well-known quotients of Fermat curves giving simple examples of non-isomorphic Riemann surfaces with many automorphisms, isomorphic (cyclic) automorphism groups, but defined over $\mathbb{Q}$, hence not Galois conjugate to each other (Theorem 2, Remark 6).

For completeness, we mention two more possibilities to obtain other Galois invariants of dessins. First one may iterate the procedure described in [JSt] leading from the hypermap group to the cartographic group, or one may pass to more general combinations $p(B)$ of the original Belyi function $B$ with other Shabat polynomials $p$ and then consider the monodromy group of $p(B)$. It is an open problem to what extent this idea leads to better Galois invariants. Secondly one may refine the Main Observation by considering not the above mentioned characters but directly the multipliers of the automorphism in their fixed points which have to be used for the calculation of the characters, see the proof of Lemma 7. But it turns out that this refinement gives no further information, see [Be] or the Proposition in Section 6. An Important Remark following this Proposition shows that, in general, characters or multipliers do not determine uniquely Riemann surfaces with many automorphisms or regular dessins. It is an open problem if in general they determine uniquely their Galois orbits as they do in the series of examples discussed in this paper. 
We obtained some useful hints by Gareth Jones, David Singerman and the referee of this paper. The second author thanks Chiba University and the Japan Society for the Promotion of Science for their hospitality in March 1998 when a first draft of this paper was written.

\section{Classification}

As already mentioned, we consider semidirect products

$$
G \cong Z_{p} \times Z_{q}
$$

of the multiplicative cyclic groups of prime order $q$ and $p \equiv 1 \bmod q$. For technical reasons we will suppose both primes to be $>3$ and $q \neq 7$. We will further suppose that $Z_{q}$ acts on the normal subgroup $Z_{p}$ by

$$
b^{-1} a b=a^{u}
$$

where $a$ and $b$ are generators of $Z_{p}$ and $Z_{q}$ respectively and $u$ denotes a fixed prime residue class of order $q$ in $(\mathbb{Z} / p \mathbb{Z})^{*}$. The conjugacy relation is in fact the essential part of the presentation, as we can see in the first point of the following Lemma (1., 3. and 6. are taken from Section 25 of [JL], the others are obvious).

\section{Lemma 1.}

1. $G$ is presented by the generators $a$ and $b$ and the relations

$$
a^{p}=b^{q}=1, \quad b^{-1} a b=a^{u} .
$$

2. For all $n, m, j \in \mathbb{N}$

$$
b^{-j} a^{n} b^{m} b^{j}=a^{n u^{j}} b^{m} .
$$

3. Denote by $S$ the subgroup generated by $u$ in the group $(\mathbb{Z} / p \mathbb{Z})^{*}$ and denote by $v_{i}, i=1, \ldots, r:=(p-1) / q$ a system of representatives of $(\mathbb{Z} / p \mathbb{Z})^{*} \bmod S$. Then $G$ splits into the $r+q$ conjugacy classes

$$
\{1\} \quad, \quad\left\{a^{v_{i} S}\right\}:=\left\{a^{v_{i} u^{j}} \mid j \in \mathbb{Z} / p \mathbb{Z}\right\}, i=1, \ldots, r
$$

and

$$
\left\{a^{m} b^{n} \mid m \in \mathbb{Z} / p \mathbb{Z}\right\}, n \not \equiv 0 \bmod q .
$$


4. The only nontrivial subgroups of $G$ are the commutator subgroup

$$
G^{\prime}=Z_{p}=\langle a\rangle
$$

and the $p$ conjugate cyclic subgroups of order $q$ generated respectively by $a^{m} b, m \in \mathbb{Z} / p \mathbb{Z}$. These subgroups form a partition of $G$.

5. $G$ can be generated by any two elements taken from two different cyclic subgroups of order $p$ or $q$.

6. $G$ has $q$ linear characters $\chi_{n}, n \in \mathbb{Z} / q \mathbb{Z}$, defined by

$$
\chi_{n}\left(a^{x} b^{y}\right)=e^{2 \pi i n y / q}
$$

and $r$ irreducible characters $\phi_{j}$ of degree $q$ defined by

$$
\begin{aligned}
\phi_{j}\left(a^{x} b^{y}\right) & =0 \quad \text { if } \quad y \not \equiv 0 \bmod q \\
\phi_{j}\left(a^{x}\right) & =\sum_{w \in S} e^{2 \pi i v_{j} w x / p} .
\end{aligned}
$$

For the next lemma recall that a triangle group $\Delta$ with signature $\langle p, q, r\rangle, p, q, r \in \mathbb{N}$, is presented by generators and relations

$$
\gamma_{0}, \gamma_{1}, \gamma_{\infty} ; \quad \gamma_{0}^{p}=\gamma_{1}^{q}=\gamma_{\infty}^{r}=\gamma_{0} \gamma_{1} \gamma_{\infty}=1
$$

and that all elements of finite order in $\Delta$ are conjugate in $\Delta$ to powers of these generators. Therefore a homomorphism $h: \Delta \rightarrow G$ has a torsion free kernel if and only if the generators of $\Delta$ are mapped to elements of the same order in $G$. These homomorphisms are easy to classify since they are uniquely determined by the images of two generators.

Lemma 2. Suppose $G$ defined as above, and suppose that

$$
h: \Delta \rightarrow G
$$

is a homomorphism with torsion free kernel. Then there are two possibilities for the signature of $\Delta$.

1. $\Delta=\langle p, q, q\rangle$. In this case there are $p(p-1)(q-1)$ different homomorphisms with torsion free kernel according to the choice of

$$
h\left(\gamma_{0}\right)=a^{m}, h\left(\gamma_{1}\right)=a^{n} b^{s}, m \in(\mathbb{Z} / p \mathbb{Z})^{*}, n \in \mathbb{Z} / p \mathbb{Z}, s \in(\mathbb{Z} / q \mathbb{Z})^{*} .
$$


2. $\Delta=\langle q, q, q\rangle$. In this case there are $p(p-1)(q-1)(q-2)$ different homomorphisms with torsion free kernel according to the choice of

$$
h\left(\gamma_{0}\right)=a^{m} b^{n}, h\left(\gamma_{1}\right)=a^{s} b^{t}, m, s \in \mathbb{Z} / p \mathbb{Z}, n, t \in(\mathbb{Z} / q \mathbb{Z})^{*},
$$

and $a^{m} b^{n}, a^{s} b^{t}$ neither lying in the same cyclic subgroup of $G$ nor having a product in $Z_{p}=\langle a\rangle($ equivalent to $n \not \equiv-t \bmod q)$.

The proof is obvious, only the last point deserves to be mentioned: $h\left(\gamma_{0}\right)$ can be chosen freely among the $p(q-1)$ elements of order $q$ in $G$, and for the choice of $h\left(\gamma_{1}\right)$ one has to avoid the $q-1$ nontrivial powers of $h\left(\gamma_{0}\right)$ and the $p-1$ possibilities leading to an $h\left(\gamma_{\infty}\right)=\left(h\left(\gamma_{0}\right) h\left(\gamma_{1}\right)\right)^{-1}$ of order $p$. For the reformulation with exponents, observe that $b^{n} a^{s} b^{t}=$ $a^{v} b^{n} b^{t}$ for some $v \in \mathbb{Z} / p \mathbb{Z}$.

Lemma 3. The automorphism group of $G$ is isomorphic to a semidirect product $(\mathbb{Z} / p \mathbb{Z})^{*} \ltimes \mathbb{Z} / p \mathbb{Z} \cong A G L_{1}(p)$. The automorphisms are determined by

$$
a \mapsto a^{k}, k \in(Z / p \mathbb{Z})^{*}, \quad b \mapsto a^{m} b, m \in \mathbb{Z} / p \mathbb{Z} .
$$

Proof. Preserving the orders, every automorphism must satisfy

$$
a \mapsto a^{k}, k \in(\mathbb{Z} / p \mathbb{Z})^{*}, \quad b \mapsto a^{m} b^{n}, m \in \mathbb{Z} / p \mathbb{Z}, n \in(\mathbb{Z} / q \mathbb{Z})^{*} .
$$

Since the defining conjugacy relation has to be preserved, we also have

$$
a^{u k}=b^{-n} a^{-m} a^{k} a^{m} b^{n}=a^{u^{n} k},
$$

see Lemma 1. But $u k \equiv u^{n} k \bmod p$ implies $u^{n-1} \equiv 1 \bmod p$, hence $n \equiv 1 \bmod q$.

\section{Corollary 1.}

1. There are $q-1$ different normal subgroups $N_{s}$ of $\Delta=\langle p, q, q\rangle$, $s \in(\mathbb{Z} / q \mathbb{Z})^{*}$, with quotient $\Delta / N_{s} \cong G$.

2. There are $(q-1)(q-2)$ different normal subgroups $N_{n, t, v}$ of $\Delta=$ $\langle q, q, q\rangle$ with quotient $\Delta / N_{n, t, v} \cong G$ where $n, t, v \in(\mathbb{Z} / q \mathbb{Z})^{*}$ satisfy the condition $n+t+v \equiv 0 \bmod q$. 
Proof and comment. It is easy to see that two homomorphisms $h_{1}, h_{2}: \Delta \rightarrow G$ have the same kernel if and only if there is an automorphism $\alpha$ of $G$ with $h_{1}=\alpha \circ h_{2}$. Therefore the respective number of different normal subgroups with quotient $G$ follows directly from the preceding lemmas. By combining the homomorphisms with suitable automorphisms, we can moreover normalize the homomorphisms in question in the following way.

For $\Delta=\langle p, q, q\rangle$ we may assume $h=: h_{s}$ with

$$
h\left(\gamma_{0}\right)=a, h\left(\gamma_{1}\right)=b^{s}
$$

for some $s \in(\mathbb{Z} / q \mathbb{Z})^{*}$ uniquely determining the kernel.

For $\Delta=\langle q, q, q\rangle$ we may assume $h=: h_{n, t, v}$ with

$$
h\left(\gamma_{0}\right)=b^{n}, \quad h\left(\gamma_{1}\right)=a b^{t}, \quad h\left(\gamma_{\infty}\right)=a^{-u^{t}} b^{v}
$$

with $n, t, v \in(\mathbb{Z} / q \mathbb{Z})^{*}, n+t+v \equiv 0 \bmod q$ following from $\gamma_{0} \gamma_{1} \gamma_{\infty}=1$. We observe that the condition $n \not \equiv-t \bmod q$ is automatically satisfied and that the normal subgroups of $\Delta$ with quotient $G$ are uniquely characterized by the triples $(n, t, v)$ as the kernels of these normalized homomorphisms.

Lemma 4. For these normal subgroups $N_{s}<1 \quad\langle p, q, q\rangle$ and $N_{n, t, v}<1$ $<q, q, q>$ we have:

1. $\Delta=\langle p, q, q\rangle$ is the normalizer of $N_{s}$ in $P S L_{2}(\mathbb{R})$.

2. $\Delta=\langle q, q, q\rangle$ is the normalizer of $N_{n, t, v}$ in $P S L_{2}(\mathbb{R})$ if $n, t, v \in$ $(\mathbb{Z} / q \mathbb{Z})^{*}$ are pairwise distinct. If not, only two of the indices can coincide, and then the normalizer is a triangle group $\bar{\Delta}=$ $<2 q, q, 2>$ containing $\Delta$ with index 2 .

Proof. In any case, the normalizer is a Fuchsian group containing $\Delta$. It is known that triangle groups have only triangle groups as possible supergroups, and using Singerman's list of inclusions [Si], one may see that the only possibilities in our situation are

$$
\begin{gathered}
<p, q, q><<<2 p, q, 2> \\
<q, q, q><<\quad<2 q, q, 2>,<q, 3,3>\subset<2 q, 3,2>.
\end{gathered}
$$


(We excluded the case $q=7$ since $\langle 7,7,7\rangle$ is exceptionally a nonnormal subgroup of $\langle 2,3,7\rangle$.)

1. Suppose that $N_{s}$ is a normal subgroup not only of $\langle p, q, q\rangle$ but also of $\langle 2 p, q, 2\rangle$. We may present the larger triangle group by generators and relations

$$
\bar{\Delta}=\left\langle\alpha, \gamma_{1}, \delta ; \quad \alpha^{2 p}=\gamma_{1}^{q}=\delta^{2}=\alpha \gamma_{1} \delta=1>\right.
$$

and relate it to the generators of $\Delta=\langle p, q, q\rangle$ by

$$
\alpha^{2}=\gamma_{0}, \quad \delta^{-1} \gamma_{1} \delta=\gamma_{\infty} .
$$

So, if we had a quotient $\bar{G} \cong \bar{\Delta} / N_{s}$ extending $G$ with index 2 , there would be an automorphism of $G$ sending

$$
h\left(\gamma_{1}\right)=b^{s} \quad \text { to } h\left(\gamma_{\infty}\right)=\left(h\left(\gamma_{0}\right) h\left(\gamma_{1}\right)\right)^{-1}=b^{-s} a^{-1}=a^{-u^{s}} b^{-s},
$$

see Lemma 1. According to Lemma 3 this would imply $s \equiv-s \bmod q$ which is clearly impossible.

2. In the case $N_{n, t, v}<1 \quad \Delta=\langle q, q, q\rangle$ we first note that $n \equiv t \equiv$ $v \bmod q$ is impossible by our assumptions $n, t, v \in(\mathbb{Z} / q \mathbb{Z})^{*}, n+t+v \equiv$ $0 \bmod q$. Consequently, there is no automorphism of $G$ giving a cyclic permutation of the generators $h\left(\gamma_{i}\right)$ (see Lemma 3) whence $N_{n, t, v}$ is never a normal subgroup of $\langle q, 3,3\rangle$ or $\langle 2 q, 3,2\rangle$ by arguments quite similar to the first case. The same arguments show that $N_{n, t, v}$ is not a normal subgroup of $\bar{\Delta}=\langle 2 q, q, 2\rangle$ if $n, t, v$ are pairwise distinct. However, the groups $N_{n, t, t}$ (say) are in fact normal subgroups of $\bar{\Delta}$ which we may see as follows. Since the order of $u$ in $(\mathbb{Z} / p \mathbb{Z})^{*}$ is odd $(=q)$, it is a quadratic residue $\bmod p$. Therefore a $w \in(\mathbb{Z} / q \mathbb{Z})^{*}$ exists satisfying

$$
w^{2} \equiv u \text { and hence } w^{q} \equiv-1 \bmod q .
$$

There is a supergroup $\bar{G}$ of $G$ of index 2 presented by

$$
\bar{G}=\left\langle a, c ; a^{p}=c^{2 q}=1, c^{-1} a c=a^{w}\right\rangle
$$

where $c^{2}=b$. As above, let $\bar{\Delta}$ be generated by $\alpha, \gamma_{1}, \delta$ of the respective orders $2 q, q, 2$ and with $\alpha^{2}=\gamma_{0}, \delta^{-1} \gamma_{1} \delta=\gamma_{\infty}$. Then one can check by 
a straightforward but lengthy calculation - playing with the relations and with $n+2 t \equiv 0 \bmod q$ - that

$$
\alpha \mapsto c^{n}, \gamma_{1} \mapsto a b^{t}, \delta \mapsto a^{-u^{-t}} c^{q}
$$

defines a homomorphism $\bar{\Delta} \rightarrow \bar{G}$ which restricts on $\Delta$ to the original homomorphism $h$ with kernel $N_{n, t, t}$.

Different normal subgroups $N_{s}$ or $N_{n, t, v}$ can be conjugate in $P S L_{2}(\mathbb{R})$ only by elements of a group containing $\Delta$ with finite index, i.e. by elements of the respective maximal triangle group. Looking at the effect on the generators we can therefore deduce the

\section{Corollary 2.}

1. Among the normal subgroups $N_{s}$ of $\Delta=\langle p, q, q\rangle$ there are $(q-1) / 2 P S L_{2}(\mathbb{R})$-conjugacy classes. For all $s \in(\mathbb{Z} / q \mathbb{Z})^{*}, N_{s}$ is conjugate to $\mathrm{N}_{-s}$.

2. The normal subgroups $N_{n, t, v}$ and $N_{m, s, w}$ of $\Delta=\langle q, q, q\rangle$ are $P S L_{2}(\mathbb{R})$-conjugate if and only if $(n, t, v)$ is a permutation of $(m, s, w)$. There are $q-1$ conjugacy classes of groups $N_{n, t, t}$ and $(q-1)(q-5) / 6$ conjugacy classes of groups $N_{n, t, v}$ with pairwise different indices.

\section{The Riemann surfaces with $p$-ramification}

Theorem 1. Let $\Delta=\langle p, q, q\rangle, G$ and $N_{s}, s \in(\mathbb{Z} / q \mathbb{Z})^{*}$, be defined as in the first case of the last section, i.e. let $N_{s}$ be the kernel of the homomorphism

$$
h_{s}: \Delta \rightarrow G \text { given by } \gamma_{0} \mapsto a, \gamma_{1} \mapsto b^{s} .
$$

Then

1. the quotient spaces $N_{s} \backslash \mathcal{H}, s \in(\mathbb{Z} / q \mathbb{Z})^{*}$, form $(q-1) / 2$ nonisomorphic compact Belyi surfaces $X_{s}$ with $X_{s} \cong X_{-s}$ for all $s$.

2. The genus of all $X_{s}$ is

$$
g\left(X_{s}\right)=\frac{1}{2}(p-1)(q-2) .
$$


3. Their automorphism group is Aut $X_{s} \cong G$.

4. Let $\zeta$ denote a fixed $q$-th root of unity $\neq 1$ and choose an integer $u$ representing its residue class $\bmod p$. Then, as an algebraic curve, $X_{s}$ has the (affine, singular) model (with $\bar{s} s \equiv 1 \bmod q$ )

$$
y^{p}=\prod_{k=1}^{q}\left(x-\zeta^{\bar{s} k}\right)^{u^{k}} .
$$

5. The curves $X_{s}, s \in(\mathbb{Z} / q \mathbb{Z})^{*}$ (or: their regular dessins) form an orbit for the action of $\mathrm{Gal} \mathbb{Q}(\zeta) / \mathbb{Q}$.

6. Their moduli field (or: minimal field of definition) is $\mathbb{Q}\left(\zeta+\zeta^{-1}\right)$.

7. The triangle group $\Delta$ has a normal subgroup $\Gamma$ of index $q$ containing all subgroups $N_{s}$ as normal subgroups of index $p$. With the homomorphisms $h_{s}$, it can be described as the preimage

$$
\Gamma:=h_{s}^{-1}\left(Z_{p}\right)=h_{s}^{-1}(<a>)
$$

or as the kernel of the homomorphism

$$
h: \Delta \rightarrow Z_{q} \text { given by } \gamma_{0} \mapsto 1, \gamma_{1} \mapsto b, \gamma_{\infty} \mapsto b^{-1} .
$$

Its quotient surface $Y=\Gamma \backslash \mathcal{H}$ is of genus 0 and the covering $Y \rightarrow \Delta \backslash \mathcal{H}$ is normal and cyclic of order $q$. It is ramified at and above two points.

Proof. 1. follows by Corollary 1.1 and Corollary 2.1 .

2. follows by the index $\left(\Delta: N_{s}\right)=p q$ (and the usual comparison of the volumes of the involved fundamental domains) as

$$
g=1+\frac{p q}{2}\left(1-\frac{1}{p}-\frac{2}{q}\right) .
$$

Another possibility is the application of the Riemann-Hurwitz formula to the $p q$-sheeted covering $X_{s} \rightarrow \mathbb{P}^{1}$ with $q$ points lying over 0 , each with ramification index $p, p$ points lying over 1 and $p$ points lying over $\infty$, each with ramification index $q$.

3. follows by Lemma 4.1 and $\Delta / N_{s} \cong G$. 
7. The existence of $\Gamma$ and its properties follow from isomorphism theorems of group theory. Note that all possible homomorphisms

$$
h_{s}=h: \Delta \rightarrow Z_{q} \text { given by } \gamma_{0} \mapsto 1, \gamma_{1} \mapsto b^{s}, \gamma_{\infty} \mapsto b^{-s}
$$

have the same kernel, so $\Gamma$ is independent of $s$. The genus of $Y$ may be computed in different ways. Since it is clear that the covering $Y \rightarrow$ $\Delta \backslash \mathcal{H} \cong \mathbb{P}^{1}$ is cyclic of order $q$ and ramified of order $q$ precisely above 1 and $\infty$ - recall the identification of $0,1, \infty$ with the fixed point orbits of $\Delta$ given in Section 1 - we have also $Y \cong \mathbb{P}^{1}$. If we denote the respective function fields of $Y$ and $\Delta \backslash \mathcal{H}$ by $\mathbb{C}(x)$ and $\mathbb{C}(t)$, the covering map (of course a Belyi function) can be explicitly given by

$$
x \mapsto x^{q}=1-t .
$$

4. The function field of $X_{s}$ is a Galois extension of $\mathbb{C}(t)$ with Galois group anti-isomorphic to $G$, and $\mathbb{C}(x)$ is the normal intermediate field fixed by the normal subgroup $Z_{p}$ generated by $a$. As a cyclic extension of $\mathbb{C}(x)$ of order $p$, we may therefore write the function field of $X_{s}$ in the form $\mathbb{C}(x, y)$ where $y^{p}$ is a rational function of $x$, uniquely determined up to taking powers with exponents prime to $p$ and up to multiplication with $p$-th powers in $\mathbb{C}(x)$. Moreover we may assume that the Belyi function $X_{s} \rightarrow \mathbb{P}^{1}$ is given by $(x, y) \mapsto x^{q}=1-t$ ramifying precisely above $t=0$ with order $p$, i.e. in the points with $x=\zeta^{k}, k \in \mathbb{Z} / q \mathbb{Z}$. Therefore we can assume that

$$
y^{p}=\prod_{k=1}^{q}\left(x-\zeta^{k}\right)^{c_{k}}
$$

with some integers $c_{k}$ not divisible by $p$. In fact, the cyclic extension only depends on the residue classes $c_{k} \bmod p$ and is invariant under multiplication with a common factor $c \in(\mathbb{Z} / p \mathbb{Z})^{*}$, so it corresponds to some point

$$
\left(c_{1}, \ldots, c_{q}\right) \in \mathbb{P}^{q-1}\left(\mathbb{F}_{p}\right)
$$

in a projective space over the finite field with $p$ elements. According to Kummer theory (see e.g. $[\mathrm{L}]$ ), this point is uniquely determined by the extension. Which point?

To decide this question we fix the $q$-th root of unity $\zeta$ and an (also 
primitive) $p$-th root of unity $\eta$ such that the action of $G \cong$ Aut $X_{1}$ on the functions satisfies

$a(y):=y \circ a^{-1}=\eta y, \quad a(x):=x \circ a^{-1}=x, \quad b(x):=x \circ b^{-1}=\zeta x$.

Now the action of $b$ on the equation for $y^{p}$ induces a cyclic shift on the place of the exponents (to simplify the argument, we can assume that all $c_{k} \equiv 1 \bmod q$, hence $\sum c_{k}$ divisible by $q$ )

$$
\prod_{k=1}^{q}\left(\zeta x-\zeta^{k}\right)^{c_{k}}=\prod_{k \bmod q}\left(x-\zeta^{k-1}\right)^{c_{k}}=\prod_{k \bmod q}\left(x-\zeta^{k}\right)^{c_{k+1}} .
$$

Since $b(y)$ is also a $p$-th root generating the field extension $\mathbb{C}(x, y) / \mathbb{C}(x)$, the shift of exponents gives the same point in $\mathbb{P}^{q-1}\left(\mathbb{F}_{p}\right)$, hence

$$
\left(c_{1}, \ldots, c_{q}\right)=\left(1, c, c^{2}, \ldots, c^{q-1}\right)
$$

for some $c \in \mathbb{F}_{p}^{*}$ of order $q$. We can moreover conclude that for a suitable integer representing the exponent, $b(y)=y^{c} g(x)$ with a rational function $g$. Using $a b=b a^{u}$ we get even $c=u$ (again a choice of an integer representing $u \bmod p$, w.l.o.g. $u \equiv 1 \bmod q$ ), and following the defining equation for $y^{p}$ we may determine $g(x)$ explicitly by

$$
g(x)^{p}=\frac{x-1}{(x-1)^{u^{q}}}=(x-1)^{1-u^{q}}
$$

(recall that $1-u^{q}$ is divisible by $p$ ). Note also that $\sum u^{k}$ is divisible by $p$ whence the covering map $X_{s} \rightarrow Y$ is unramified over $x=\infty$.

Finally one can replace $\zeta$ by any other primitive $q$-th root of unity $\zeta^{\bar{s}}$ corresponding to other choices of the generator of $Z_{q}$ or to other choices of the homomorphism $h_{s}$.

5. follows from the equation given in 4 .

6. may be seen using $X_{s} \cong X_{-s}$ or also by a more direct argument involving the equations given in 4.: recall that $\sum u^{k}=p N$ for an integer $N$ and that we can assume $u \equiv 1 \bmod q$ and therefore $\sum \bar{s} k u^{k} \equiv$ $0 \bmod q$. Now write the equation for $X_{-s}$ as

$$
y^{p}=\prod\left(x-\zeta^{-\bar{s} k}\right)^{u^{k}}=x^{p N} \prod\left(\zeta^{\bar{s} k}-\frac{1}{x}\right)^{u^{k}}
$$


and write again $x$ for $1 / x$ and $y$ for $\pm y / x^{N}$ to deduce the equation for $X_{s}$.

Remarks. 1. We take the opportunity to point out a possible misunderstanding in Remark 4 of [Wo1]. Recall that a Belyi pair means a pair $(U, \beta)$ of a Belyi surface $U$ with a Belyi function $\beta$ on it. Two Belyi pairs $(U, \beta)$ and $(V, \phi)$ are called isomorphic if there are isomorphisms

$$
f: U \rightarrow V, \mu: \mathbb{P}^{1} \rightarrow \mathbb{P}^{1} \text { with } \phi \circ f=\mu \circ \beta
$$

(weakly isomorphic in the terminology of $[\mathrm{CG}]$ ). As in the definition of moduli fields of Belyi surfaces, let $H$ be the subgroup of $\mathrm{Gal} \overline{\mathbb{Q}} / \mathbb{Q}$ consisting of all $\sigma$ which conjugate the Belyi pair $(U, \beta)$ into an isomorphic Belyi pair $\left(U^{\sigma}, \beta^{\sigma}\right)$. Then call the fixed field $M(U, \beta)$ of $H$ the moduli field of $(U, \beta)$. It is automatically contained in every common field of definition of $U$ and $\beta$. For a Riemann surface with many automorphisms $N \backslash \mathcal{H}=X, N$ a normal subgroup of a triangle group $\Delta$, and its Belyi function $B: X \rightarrow \mathbb{P}^{1}=\Delta \backslash \mathcal{H}$ it is true that the moduli fields $M(X)$ and $M(X, B)$ coincide and that $X$ can be defined over $M(X)$. But this is possibly not true for $B$ because $B$ is unique (canonical) only up to automorphisms of $\mathbb{P}^{1}$ exchanging $0,1, \infty$. In cases where $\Delta$ is not maximal, i.e. if the same order occurs at non-equivalent fixed points it may happen that a Galois conjugation in $\mathrm{Gal} \overline{\mathbb{Q}} / M(X)$ exchanges the fixed points of $\Delta$, hence $B \neq B^{\sigma}$ (in the terminology of [CG], the Belyi pairs are not strongly isomorphic). In that case, $B$ can only be defined over a field containing $M(X)$ with index 2,3 or 6 . This phenomenon occurs precisely for our Belyi surfaces $X_{s}$ where the isomorphism to $X_{-s}$ corresponds to an exchange of 1 and $\infty$.

2. For Theorem 1 the primes $q=3$ and 7 are admitted, but for $q=3$ we obtain curves which will be treated in the next section under the name $Y_{1, t, t^{2}}$ (the other prime here called $p$ will there be called $q$ ).

3. Since it was not too difficult, we described here the Galois action using the explicit equations. This can be avoided using fixed points and multipliers on canonical models, see Section 5.

\section{A well-known normal subcovering}

Now we will concentrate on those cases where $G$ is a quotient of $\Delta=$ $<q, q, q\rangle$. In this section we consider the normal subcovering of 
$X_{n, t, v} / \mathbb{P}^{1}$ coming from the Fuchsian group $\Gamma_{n, t, v}:=h_{n, t, v}^{-1}\left(Z_{p}\right)$ where $h_{n, t, v}$ denotes the normalized homomorphism $\Delta \rightarrow G$ introduced in the proof of Corollary 1.2. As always, $n, t, v \in(\mathbb{Z} / q \mathbb{Z})^{*}$ with $n+t+v \equiv$ $0 \bmod q$. We already know that the isomorphism classes of $X_{n, t, v}$ depend on $n, t, v$ up to permutation only.

Lemma 5. As $n, t, v$ let $m, s, w \in(\mathbb{Z} / q \mathbb{Z})^{*}$ with $m+s+w \equiv 0 \bmod q$ and call

$$
(n, t, v) \sim(m, s, w)
$$

if and only if these triples coincide up to permutation and multiplication by a common factor $c \in(\mathbb{Z} / q \mathbb{Z})^{*}$. This is an equivalence relation. We denote the equivalence classes by $[n, t, v]$.

1. The only homomorphisms of $\Delta=\langle q, q, q\rangle$ onto $Z_{q}=\langle b\rangle$ with torsion-free kernel are given by

$$
f_{n, t, v}: \gamma_{0} \mapsto b^{n}, \gamma_{1} \mapsto b^{t}, \gamma_{\infty} \mapsto b^{v}
$$

with $n, t, v \in(\mathbb{Z} / q \mathbb{Z})^{*}, n+t+v \equiv 0 \bmod q$.

2. The kernel of $f_{n, t, v}$ is $\Gamma_{n, t, v}$.

3. There is a bijection between the $P S L_{2}(\mathbb{R})$-conjugacy classes of $\Gamma_{n, t, v}$ and the equivalence classes $[n, t, v]$.

4. The normalizer of $\Gamma_{n, t, t}$ in $P S L_{2}(\mathbb{R})$ is $\bar{\Delta}=\langle 2 q, q, 2\rangle$. If there is a $t \in(\mathbb{Z} / q \mathbb{Z})^{*}$ of order 3 and with $1+t+t^{2} \equiv 0 \bmod q$ (which happens if and only if $q \equiv 1 \bmod 3$ ) then the normalizer of $\Gamma_{1, t, t^{2}}$ is $\bar{\Delta}=\langle q, 3,3\rangle$ containing $\Delta$ as normal subgroup of index 3 . In all other cases $\Delta$ is the normalizer of $\Gamma_{n, t, v}$.

Proof. 1. follows from the relation between the generators of $\Delta$.

2. The homomorphism $f_{n, t, v}$ is a combination of $h_{n, t, v}$ with the canonical projection of $G$ onto the factor group $G / Z_{p} \cong Z_{q} \cong\langle b\rangle$.

3. If $c \in(\mathbb{Z} / q \mathbb{Z})^{*}, f_{c n, c t, c v}$ is $f_{n, t, v}$, followed by the automorphism $Z_{q} \rightarrow Z_{q}$ given by $b \mapsto b^{c}$, whence the kernels are invariant under multiplication of the indices with common factors. The invariance of the $P S L_{2}(\mathbb{R})$-conjugacy class under permutations of the indices follows as in Corollary 2.2 by the fact that the generators of $\Delta$ can be permuted under the action of $\bar{\Delta}=\langle 2 q, 3,2\rangle$. The assumptions on $p$ and $q$ 
guarantee that no other conjugations between the $\Gamma_{n, t, v}$ are possible. 4. If $t=v$ the homomorphism $f_{n, t, v}$ clearly extends to a homomorphism of $\langle 2 q, q, 2\rangle$ onto a group $Z_{2 q}$ containing $Z_{q}=\langle b\rangle$ (exercise). In the second case mentioned, there is an index 3 extension $H$ of $Z_{q}$ containing $Z_{q}$ as normal subgroup and a non-normal subgroup $\langle d\rangle$ of order 3 acting on $Z_{q}$ via

$$
d^{-1} b d=b^{t} .
$$

Then $f_{1, t, t^{2}}$ is extendable to a homomorphism of $\langle q, 3,3\rangle$ onto this semidirect product $Z_{q} \rtimes Z_{3}$. Observe that the conjugation by one of the generators of $\bar{\Delta}$ permutes the generators of $\Delta$.

It is easy to check that not both cases can occur at the same time, therefore $\Delta$ is the normalizer in all other cases.

Theorem 2. Let $n, t, v$ be as above and define $Y_{n, t, v}:=\Gamma_{n, t, v} \backslash \mathcal{H}$. Then we have

1. $Y_{n, t, v}$ is a Belyi surface of genus $(q-1) / 2$ and with many automorphisms. Its Belyi function

$$
Y_{n, t, v} \rightarrow \Delta \backslash \mathcal{H} \cong \mathbb{P}^{1}
$$

is a normal cyclic covering of degree $q$ and with ramifications above $0,1, \infty$ of order $q$.

2. The automorphism group is

Aut $Y_{n, t, v} \cong Z_{q}$ if $n, t, v$ are pairwise distinct and not equivalent to $\left(1, t, t^{2}\right)$ with $t^{3} \equiv 1,1+t+t^{2} \equiv 0 \bmod q$, and in these cases we have

$$
\begin{aligned}
& \text { Aut } Y_{1, t, t^{2}} \cong Z_{q} \rtimes Z_{3}, \\
& \text { Aut } Y_{n, t, t} \cong Z_{2} \times Z_{q} .
\end{aligned}
$$

The surfaces $Y_{n, t, t}$ are hyperelliptic.

3. $Y_{n, t, v} \cong Y_{m, s, w}$ if and only if $(n, t, v) \sim(m, s, w)$.

4. $Y_{n, t, v}$ has the (affine, singular) model over $\mathbb{Q}$

$$
y^{q}=x^{n}(x-1)^{t} .
$$


Proof. 1., 2. and 3. follow almost directly from Lemma 5. The genus can be again computed by Riemann-Hurwitz, and in the $t=v$ case one can see that in fact $Y_{n, t, t} / Z_{2} \cong \mathbb{P}^{1}$ or transform the equation of part 4 . into an equation $u^{2}=\ldots$. The shape of the equation can be guessed as follows. The function field $\mathbb{C}(y, x)$ of $Y_{n, t, v}$ is a cyclic extension of the function field $\mathbb{C}(x)$ of $\mathbb{P}^{1}$, and the ramification shows that we can assume $y^{q}=x^{\mu}(x-1)^{\nu}$. To find the correct exponents, choose $y$ such that $b(y)=\zeta y, \zeta$ the primitive $q$-th root of unity for which $\gamma_{0}$ acts in its fixed points on local branches of $\sqrt[q]{x}$ like multiplication by $\zeta$, and so on for $\gamma_{1}, \gamma_{\infty}$.

Remarks. 4. The preceding proof follows a suggestion of Gareth Jones. Another possibility to distinguish the different non-isomorphic surfaces $Y_{n, t, v}$ is the fact that their Jacobians have complex multiplication by the cyclotomic field $\mathbb{Q}(\zeta)$ but that their isogeny class can be distinguished by the CM type. Moreover, the different CM types correspond bijectively to the equivalence classes $[n, t, v]$, see $[\mathrm{KR}]$, so we can apply Torelli's theorem to distinguish the curves. 5. In particular, $[\mathrm{KR}]$ gives the following additional information. Non-isomorphic surfaces $Y_{n, t, v}$ are not Galois-conjugate but the automorphism groups (or: hypermap groups for their canonical regular dessin) coincide - at least in general for $q \geq 17$, see below - so they are incomplete as Galois invariants. However, here the curves may be distinguished by the Main Observation: the eigenspace decomposition of the usual representation

$$
\rho: \alpha \mapsto\left(\omega \mapsto \omega \circ \alpha^{-1}\right), \alpha \in Z_{q},
$$

on the vector space of holomorphic differentials on $Y_{n, t, v}$ corresponds bijectively up to isomorphism to the CM type of $\operatorname{Jac} Y_{n, t, v}$. Therefore, the non-isomorphic surfaces can be distinguished by the trace of $\rho(b)$. We will come back to this question in Section 6 .

6. For $q=5$ there is only one equivalence class of triples corresponding to the well-known hyperelliptic curve $y^{5}=x(x-1)$ or $u^{2}=v^{5}-1$. For $q=11$ there is one hyperelliptic and one ordinary triple. For $q=13$ there are three inequivalent triples of all types, namely

$$
[1,6,6],[1,2,10],[1,3,9] \quad(g=6)
$$

The Jacobian of $Y_{1,3,9}$ is a product of three isogenous abelian varieties of dimension 2, and the curve is a cover of another curve of genus 2 , 
obtained by taking the quotient by the factor $Z_{3}$ of the automorphism group. First for $q=17$ we have two ordinary triples (and one hyperelliptic, of course), namely

$$
[1,8,8],[1,2,14],[1,3,13] \quad(g=8) .
$$

7. Let us shed some light on the excluded case $q=7$. Here we are in genus 3 and have a hyperelliptic curve $Y_{1,3,3}$ and a non-hyperelliptic curve $Y_{1,2,4}$. By the techniques going back to Shimura and Taniyama and extensively used by Koblitz and Rohrlich [KR], the Jacobian of the latter curve is isogenous to an elliptic curve with complex multiplication by $\mathbb{Q}(\sqrt{-7})$. But here $Z_{7}$ or $Z_{7} \rtimes Z_{3}$ are far from being the true automorphism group. As a consequence of the exceptional situation $<7,7,7>\subset<2,3,7>$ the full automorphism group is $P S L_{2}\left(\mathbb{F}_{7}\right)$ and the curve is isomorphic to Klein's quartic.

\section{The Riemann surfaces without $p$-ramification}

Theorem 3. Let $\Delta=\langle q, q, q\rangle, G$ and $N_{n, t, v}$ be defined as in the second case of Corollary 2, i.e. with $n, t, v \in(\mathbb{Z} / q \mathbb{Z})^{*}, n+t+v \equiv$ $0 \bmod q, N_{n, t, v}$ the kernel of the homomorphism

$h_{n, t, v}=h \quad$ defined by $h\left(\gamma_{0}\right)=b^{n}, h\left(\gamma_{1}\right)=a b^{t}, h\left(\gamma_{\infty}\right)=a^{-u^{t}} b^{v}$.

Then

1. the quotient spaces $N_{n, t, v} \backslash \mathcal{H}$ are compact Belyi surfaces $X_{n, t, v}$ of genus

$$
g=1+\frac{1}{2} p(q-3) .
$$

$X_{n, t, v}$ is isomorphic to $X_{m, s, w}$ if and only if $(n, t, v)$ is a permutation of $(m, s, w)$.

2. Their automorphism group is

$G \quad$ if the indices are pairwise distinct

$$
\bar{G}=Z_{p} \rtimes Z_{2 q} \quad \text { for } \quad X_{n, t, t}
$$

(for the definition of $\bar{G}$ see the proof of Lemma 4). 
3. For $(n, t, v)$ fixed, the surfaces $X_{s n, s t, s v}, s \in(\mathbb{Z} / q \mathbb{Z})^{*}$, form the orbit under the action of $\mathrm{Gal} \overline{\mathbb{Q}} / \mathbb{Q}$. More precisely, for $\zeta:=e^{2 \pi i / q}$ and $\sigma(\zeta)=\zeta^{\bar{s}}, \bar{s} s \equiv 1 \bmod q$, we have

$$
X_{n, t, v}^{\sigma} \cong X_{s n, s t, s v}
$$

4. Under the Galois action, the isomorphism classes form

- one orbit consisting of all $q-1$ surfaces $X_{n, t, t}$, all with moduli field (= minimal field of definition) $\mathbb{Q}(\zeta)$,

- one orbit consisting of all $(q-1) / 3$ non-isomorphic surfaces $X_{s, s t, s t^{3}}$ if $q \equiv 1 \bmod 3$ and $t^{3} \equiv 1 \bmod q, 1+t+t^{2} \equiv$ $0 \bmod q$. In this case, their moduli field is the fixed field $\mathbb{K}$ of $\tau: \zeta \mapsto \zeta^{t}$ in the cyclotomic field $\mathbb{Q}(\zeta)$.

- All other orbits are of length $q-1$ and consist of surfaces $X_{s n, s t, s v}, s \in(\mathbb{Z} / q \mathbb{Z})^{*}$. Here the moduli field is again $\mathbb{Q}(\zeta)$.

Proof. 1. is clear by the definition of the normal torsion-free subgroups $N_{n, t, v}$ and a genus computation analogous to that of Theorem 1:

$$
g=1+\frac{p q}{2}\left(1-\frac{3}{q}\right) .
$$

The isomorphisms between the different Belyi surfaces of this type are determined by Corollary 2.2 .

2. follows from Lemma 4.2 and its proof.

3. If $X_{n, t, v}$ and $X_{m, s, w}$ belong to the same Galois orbit, then clearly also their respective quotients $Y_{n, t, v}$ and $Y_{m, s, w}$ by the unique normal subgroup $Z_{p}<\mid G$. Theorem 2 shows that these are Galois conjugate if and only if they are isomorphic if and only if their index triples are equivalent. So $X_{n, t, v}$ and $X_{m, s, w}$ belong to the same Galois orbit at most if $(n, t, v) \sim(m, s, w)$. To see that this condition is also sufficient, we follow the ideas of [St2] and need some preparation. Recall that for $\alpha \in G$ with fixed point $P \in X_{n, t, v}$ there is a local variable $z$ with $z(P)=0$ such that the action of $\alpha$ locally is described by $z \mapsto \xi z$ for some root of unity $\xi$. We will call this $\xi$ (uniquely determined by $P$ and $\alpha$ ) the multiplier of $\alpha$ in $P$. 


\section{Lemma 6.}

1. For the action of $G$ on $X_{n, t, v}$ there are $3 p$ fixed points of order $q$.

2. Every element $a^{m} b^{n}$ of order $q, m \in \mathbb{Z} / p \mathbb{Z}, n \in(\mathbb{Z} / q \mathbb{Z})^{*}$, has three fixed points.

3. In its three fixed points, $b$ acts with multipliers $\zeta^{\bar{n}}, \zeta^{\bar{t}}, \zeta^{\bar{v}}$ where $\bar{n}, \bar{t}, \bar{v}$ denote the inverses of $n, t, v \in(\mathbb{Z} / q \mathbb{Z})^{*}$ respectively, i.e. with $\bar{n} n \equiv \bar{t} t \equiv \bar{v} v \equiv 1 \bmod q$.

Proof of Lemma 6. 1. follows by a simple counting argument: The surface is triangulated by $p q$ images of $\Delta$-fundamental domains. To each of these fundamental domains belong three fixed points of order $q$, but every such fixed point belongs to $q \Delta$-fundamental domains. (David Singerman indicated to us another possible argument using results of Macbeath $[\mathrm{Mc}]$.)

2. In $G$, there are precisely $p$ conjugate subgroups of order $q$, see Lemma 1.4. Two different such subgroups can be generated by $a^{m} b, a^{k} b$ with $m \not \equiv k \bmod p$. If $P$ were a common fixed point for both it would also be a fixed point for $a$ by consequence of

$$
b^{-1} a^{-k+m} b(P)=P
$$

and the defining relations in $G$. But $a$ acts fixed-point free on the surface, whence every subgroup of order $q$ has a fixed point triple, and these triples form in fact an orbit under the action of $Z_{p}$.

3. On the universal covering $\mathcal{H}$ of $X_{n, t, v}$ the generators $\gamma_{0}, \gamma_{1}, \gamma_{\infty}$ of $\Delta$ act in their respective fixed points with multiplier $\zeta$. Since $h\left(\gamma_{0}\right)=b^{n}$, the fixed point of $\gamma_{0}$ has to be applied by the universal covering map $\mathcal{H} \rightarrow X_{n, t, v}$ on one of the fixed points of $b$, and there $b$ must have the multiplier $\zeta^{\bar{n}}$. The same argument applies in the fixed points of $\gamma_{1}$ and $\gamma_{\infty}$ if we observe that e.g. $a b^{t}$ is conjugate to $b^{t}$ (see Lemma 1.3) and has therefore the same multipliers in its fixed points.

Proof of Theorem 3, continued. Now we observe that no automorphism group of our surfaces in question contains $Z_{2}$ as normal subgroup whence they are not hyperelliptic. Therefore the canonical model exists, constructed as the image of the following embedding. Let $\omega_{1}, \ldots, \omega_{g}$ be 
a basis of the vector space of holomorphic differentials on $X_{n, t, v}$. Then the map

$$
X_{n, t, v} \rightarrow \mathbb{P}^{g-1}: P \mapsto\left(\omega_{1}(P), \ldots, \omega_{g}(P)\right)
$$

is a well-defined holomorphic embedding, so we may now identify $X_{n, t, v}$ with its image (automatically a projective algebraic curve by Chow's theorem). By [St1] it has the following remarkable property. The representation introduced for the Main Observation in Section 1

$$
\psi: G \rightarrow G L\left(H^{0}\left(X_{n, t, v}, \Omega\right)\right)
$$

on the vector space of holomorphic 1-differentials defined by

$$
\psi(\alpha): \omega \mapsto \omega \circ \alpha^{-1}
$$

induces an action of $\psi(G) /\{ \pm 1\}$ as subgroup of $P G L_{g}(\mathbb{C})$ on the canonical model. Now let $P$ be a fixed point for $\alpha \in G$ on this canonical model with multiplier $\xi$. As in [St2], $P \in \mathbb{P}^{g-1}$ can be represented as an eigenvector for $\psi(\alpha)$ with eigenvalue $\xi^{-1}$. In our particular case, $\psi\left(b^{-1}\right)$ has three eigenvectors giving fixed points of $X_{n, t, v}$ and belonging to eigenvalues $\zeta^{\bar{n}}, \zeta^{\bar{t}}, \zeta^{\bar{v}}$, see Lemma 6 , and these eigenvalues uniquely determine the isomorphism class of $X_{n, t, v} .\left(\psi\left(b^{-1}\right)\right.$ may have more eigenvectors, in particular for other eigenvalues, but these do not give points of the canonical model.)

Let $\sigma \in \mathrm{Gal} \overline{\mathbb{Q}} / \mathbb{Q}$ be any Galois conjugation, tacitly assumed to be extended to a field automorphism of $\mathbb{C}$. As explained in Section 1, $\sigma$ maps $X_{n, t, v}$ to a Galois conjugate curve $X_{n, t, v}^{\sigma}$ which is again a canonical model because the basis $\omega_{1}, \ldots, \omega_{g}$ is transformed into a basis $\omega_{1}^{\sigma}, \ldots, \omega_{g}^{\sigma}$ of holomorphic differentials on $X_{n, t, v}^{\sigma}$. The representation matrices $\psi(\alpha)$ are transformed by coefficient-wise conjugation into representation matrices $\psi(\alpha)^{\sigma}$. In particular, the fixed points $P$ of $b^{-1}$ on $X_{n, t, v}$ become fixed points $P^{\sigma}$ of $b^{-1}$ on $X_{n, t, v}^{\sigma}$, now corresponding to eigenvectors of $\psi\left(b^{-1}\right)^{\sigma}$ for eigenvalues $\sigma\left(\zeta^{\tilde{n}}\right), \sigma\left(\zeta^{\bar{t}}\right), \sigma\left(\zeta^{\bar{v}}\right)$. There is a unique $s \in(\mathbb{Z} / q \mathbb{Z})^{*}$ satisfying $\sigma(\zeta)=\zeta^{\bar{s}}$ for $\bar{s} \in(\mathbb{Z} / q \mathbb{Z})^{*}, \bar{s} s \equiv$ $1 \bmod q$. Since $X_{n, t, v}^{\sigma}$ is uniquely determined up to isomorphism by the eigenvalues $\zeta^{\bar{s} \bar{n}}, \zeta^{\bar{s} \bar{t}}, \zeta^{\bar{s} \bar{v}}$, the only choice of the Galois conjugated surface is $X_{s n, s t, s v}$.

4. Observe that $\sigma \in \mathrm{Gal} \overline{\mathbb{Q}} / \mathbb{Q}$ with $\sigma(\zeta)=\zeta^{\bar{s}}, s \in(\mathbb{Z} / q \mathbb{Z})^{*}$ transforms 
$X_{n, t, v}$ into an isomorphic surface if and only if $(s n, s t, s v)$ is a permutation of $(n, t, v)$.

Remark 8. Remark 1 applies vice versa to the surfaces $X_{s, s t, s t^{2}}$ and their canonical Belyi functions $B$ if $t^{3} \equiv 1,1+t+t^{2} \equiv 0 \bmod q$. Here $B$ is definable over the cubic extension $\mathbb{Q}(\zeta)$ of its moduli field only.

\section{Representations}

Now we consider the representation $\psi_{k}$ on holomorphic k-differentials introduced for the Main Observation in Section 1. By Eichler's trace formula (see [FK]) the trace $\operatorname{tr}\left(\psi_{k}(\alpha)\right)$ of an automorphism $\alpha$ can be easily calculated by studying the fixed point behaviour of $\alpha$ on $X$. For $\alpha \neq 1$ let be Fix $(\alpha)=\left\{P_{1}, \ldots P_{t}\right\}$ the possibly empty set of all fixed points of $\alpha$ and $\left(\zeta_{P_{1}}, \ldots, \zeta_{P_{t}}\right)$ be the (unordered) collection of all multipliers of $\alpha$, i.e. the primitive roots of unity of the same order as $\alpha$, which describe the local action of $\alpha$ at $P_{i}, i=1, \ldots, t$. That simply says that we can choose a chart around $P_{i}$ such that $P_{i}$ is mapped to 0 and $\alpha$ is described by $z \mapsto \zeta_{P_{i}} z$. Then Eichler's trace formula states:

$$
\operatorname{tr}\left(\psi_{k}(\alpha)\right)=\left\{\begin{array}{cc}
\sum_{P \in \text { Fix }(\alpha)} \frac{\zeta_{P}^{-k}}{1-\zeta_{p}^{-1}}+\delta_{1 k} & \alpha \neq 1 \\
(2 k-1)(g-1)+\delta_{1 k} & \alpha=1 .
\end{array}\right.
$$

In the above formula the empty sum is taken to be zero and $\delta$ is the Kronecker symbol. Summarizing this one can state that the fixed point behaviour of $\alpha$ yields enough information to calculate the traces of all $\psi_{k}(\alpha)$. Less obvious is that also the converse is true.

Proposition. Let $\left(\operatorname{tr}\left(\psi_{k}(\alpha)\right)\right)_{k \in \mathbb{N}}$ be given. Then the multiplier data $\left(\zeta_{P_{1}}, \ldots, \zeta_{P_{t}}\right)$ of $\alpha$ are uniquely determined.

Proof. Consider the function

$$
f_{\alpha}(z):=\sum_{k \in \mathbb{N}} \operatorname{tr}\left(\psi_{k}(\alpha)\right) z^{k}
$$


which is defined for $|z|<1$ but which has a meromorphic continuation on $\mathbb{C}$ :

$$
\begin{aligned}
f_{\alpha}(z) & =z+\sum_{k \in \mathbb{N}} \sum_{P \in F i x(\alpha)} \frac{\zeta_{P}^{-k}}{1-\zeta_{P}^{-1}} z^{k}=z+\sum_{P \in F i x(\alpha)} \frac{1}{1-\zeta_{P}^{-1}} \sum_{k \in \mathbb{N}}\left(\zeta_{P}^{-1} z\right)^{k} \\
& =z+\sum_{P \in F i x(\alpha)} \frac{1}{1-\zeta_{P}^{-1}} \frac{\zeta_{P}^{-1} z}{1-\zeta_{P}^{-1} z} .
\end{aligned}
$$

Therefore we see that $\alpha$ rotates like $\zeta_{P}$ at a fixed point $P$ iff $f_{\alpha}$ has a pole at $z=\zeta_{P}$. The residue

$$
\zeta_{p}^{-1}\left(1-\zeta_{p}^{-1}\right) \operatorname{Res}\left(f_{\alpha}(z), z=\zeta_{P}\right)
$$

gives the number of points with the same multiplier $\zeta_{P}$ with respect to $\alpha$. This shows the statement.

This result also appears in $[\mathrm{Be}]$.

Important Remark. In the Main Observation of Section 1 we have seen that the equivalence class of $\left(t r \psi_{k}\right)_{k \in \mathbb{N}}$ is an invariant of the surface $X$ and the equivalence classes of the Galois orbit $\left(\operatorname{tr} \psi_{k}^{\sigma}\right)_{k \in \mathbb{N}}$ with $\sigma \in \mathrm{Gal} \overline{\mathbb{Q}} / \mathbb{Q}$ is an invariant of the Galois orbit of $X$. In the following we will show how to calculate these and that they are finer invariants as the previously known invariants. However, the equivalence class of $\left(t r \psi_{k}\right)_{k \in \mathbb{N}}$ does not determine the surface $X$, i.e. the dessin uniquely. This is easiest seen by taking the elliptic curve $E: y^{2}=x(x-1)(x-\lambda)$ with $\lambda=\sqrt[3]{1 / 2} \in \mathbb{R}$. A Belyi function is given by $\beta(x)=4 x^{3}\left(1-x^{3}\right)$ and we consider the smallest regular cover $X$ of $(E, \beta)$. Using the known isomorphism between the monodromy group of $\beta$ (the cartographic group of the dessin) and the automorphism group Aut $X$ (see [Wo2], Thm. 2 ), one can use computer algebra systems like [GAP] to show that Aut $X$ has order $2^{6} \cdot 3^{2}=576$ and must therefore be solvable. The ramification structure of $(E, \beta)$ is $(4,2,2,2,2)$ for the points above 1 and $(6,2,1,1,1,1)$ for the points above 0 . The unique point above $\infty$ has therefore ramification order (12). Below we have the corresponding dessin of $(E, \beta)$ where opposite sides have to be identified. 


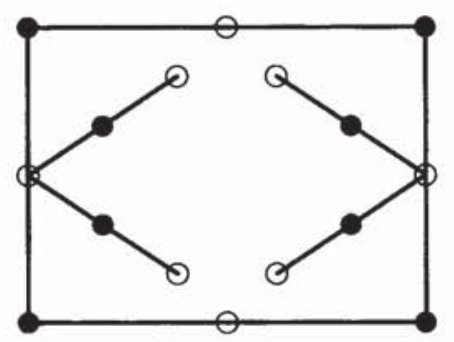

$(E, \beta)$

$E$ turns out to be the unique intermediate elliptic curve between $X$ and Aut $X \backslash X$ with the above ramification structure. Therefore an element $\sigma \in \mathrm{Gal} \overline{\mathbb{Q}} / \mathbb{Q}$ which stabilizes $X$, i.e. $X \cong X^{\sigma}$ has to stabilize $E$. Now choose $\sigma$ to be trivial on the maximal abelian extension of $\mathbb{Q}$ and nontrivial on $\mathbb{Q}(\sqrt[3]{2})$. Such a $\sigma$ would stabilize all traces but could not stabilize $X$.

Now to apply Eichler's trace formula, we have to calculate the fixed point behaviour of a given group element $\alpha$. From the point of view of dessins we have 3 special group elements $\alpha_{0}, \alpha_{1}, \alpha_{\infty}$ generating the cartographic group, which in the case of regular dessins is isomorphic to the automorphism group of the surface $X$. They are homomorphic images of the generators $\gamma_{0}, \gamma_{1}, \gamma_{\infty}$ of $\Delta$ and can directly be read off the dessin. The white and black vertices and the face centers of the dessin can be thought to be represented by left cosets $g U_{i}$ of the cyclic groups $U_{0}, U_{1}, U_{\infty}$ generated by $\alpha_{0}, \alpha_{1}, \alpha_{\infty}$ respectively: as in the proof of Lemma $6, X$ is triangulated by $\mid$ Aut $X \mid$ images of a fundamental domain $D$ for $\Delta$. We can choose $D$ such that e.g. $\left\langle\gamma_{0}\right\rangle D$ is a neighbourhood of the fixed point $z_{0}$ of $\gamma_{0}$ and $\gamma<\gamma_{0}>D$ a neighbourhood of $\gamma\left(z_{0}\right)$ for all $\gamma \in \Delta$. Since these points project to the white vertices on $X$ they correspond bijectively to the left cosets $g U_{0}$ for Aut $X \cong \Delta / \Gamma$ and $g=\gamma \Gamma$. In this sense a fixed point of $\alpha$ is a coset which is stabilized by $\alpha$ and must therefore satisfy an equation

$$
\alpha g=g \alpha_{i}^{k}
$$

where $\mathrm{g}$ is a representative of the stabilized coset $g U_{i}, i=0,1, \infty$. It is now clear that we can calculate not only the number of fixed points 
of $\alpha$ but also the multiplier: it is $\zeta_{i}^{k}$ if $\alpha_{i}$ has the multiplier $\zeta_{i}$ and $\alpha$ satisfies the above equation. As these are the only information the trace formula requires to calculate $\operatorname{tr}\left(\psi_{k}(\alpha)\right)$ we are done. Below we exhibit these calculations for the curves $X_{s}, X_{n, t, v}$.

Lemma 7. Let $G, a, b, \zeta, \eta, X_{s}, X_{n, t, v}$ be defined as in Theorems 1 and 3 and their proofs, $\bar{n}, \bar{t}, \bar{v}$ as in Lemma 6, and as in Lemma 1 let $S$ denote the subgroup of $(\mathbb{Z} / p \mathbb{Z})^{*}$ generated by $u$. For all $m \in$ $\mathbb{Z} / p \mathbb{Z}, x \in(\mathbb{Z} / p \mathbb{Z})^{*}, y \in(\mathbb{Z} / q \mathbb{Z})^{*}$, we have

1. on $X_{s}$

$$
\begin{aligned}
& \operatorname{tr} \psi(1)=\frac{1}{2}(p-1)(q-2), \operatorname{tr} \psi\left(a^{m} b^{-y}\right)=0, \\
& \operatorname{tr} \psi\left(a^{-x}\right)=1+\sum_{w \in S} \frac{\eta^{x w}}{1-\eta^{x w}}
\end{aligned}
$$

2. on $X_{n, t, v}$

$$
\begin{gathered}
\operatorname{tr} \psi(1)=1+\frac{1}{2} p(q-3), \quad \operatorname{tr} \psi\left(a^{-x}\right)=1, \\
\operatorname{tr} \psi\left(a^{m} b^{-y}\right)=1+\frac{\zeta^{\bar{n} y}}{1-\zeta^{\bar{n} y}}+\frac{\zeta^{\bar{t} y}}{1-\zeta^{\bar{t} y}}+\frac{\zeta^{\bar{v} y}}{1-\zeta^{\bar{v} y}} .
\end{gathered}
$$

The proof for the surfaces $X_{n, t, v}$ follows the program explained above and is essentially carried out in Lemma 6 : recall that by Lemma 1.3, $a^{m} b^{-y}$ is conjugate to $b^{-y}$ and therefore has the same multipliers in its three fixed points, and recall that $a$ acts without fixed points. For the first part of the Lemma consider first the quotient $Y=Z_{p} \backslash X_{s}=$ $\langle a\rangle \backslash X_{s}$ of genus 0 treated in Theorem 1.7. There, $b$ has two fixed points with multipliers $\zeta$ and $\zeta^{-1}$. Each of them lifts to $p$ fixed points on $X_{s}$ with the same multiplier (the covering $X_{s} \rightarrow Y$ is ramified in other points). Clearly, the generators $a^{m} b, m \in \mathbb{Z} / p \mathbb{Z}$ of the $p$ subgroups of order $q$ have two fixed points with multipliers $\zeta$ and $\zeta^{-1}$, respectively. Taking $(-y)$-th powers, Eichler's trace formula gives for all $m \in \mathbb{Z} / p \mathbb{Z}, y \in(\mathbb{Z} / q \mathbb{Z})^{*}$

$$
\operatorname{tr} \psi\left(a^{m} b^{-y}\right)=1+\frac{\zeta^{y}}{1-\zeta^{y}}+\frac{\zeta^{-y}}{1-\zeta^{-y}}=0 .
$$


By the construction of $X_{s}$, the element $a$ has $q$ fixed points given by the coordinates $x=\zeta^{k}, y=0$ on the model of Theorem 1.4. or equivalently by the $\langle a\rangle-$ cosets given by the $q$ different $b$ powers $b, b^{2}, \ldots, b^{q}$ as representatives of these cosets. Now $a$ satisfies the equation

$$
a b^{k}=b^{k} a^{u^{k}},
$$

which gives the multipliers $\eta^{u}, \eta^{u^{2}}, \ldots, \eta^{u^{9}}$ and we can in fact apply Eichler's trace formula.

The behaviour of $t r \psi$ under Galois actions and the decomposition of $\psi$ in irreducible components by means of Eichler's trace formula is not obvious. It becomes easier if we state it in a different but equivalent way, more in the spirit of Chevalley's and Weil's paper [CW]. For a real number $r$ let $\langle r\rangle=r-[r]$ denote the fractional part and as always $\eta=e^{2 \pi i / p}$.

Lemma 8. Let $m_{1}, \ldots, m_{k}$ and $\bar{m}_{1}, \ldots, \bar{m}_{k} \in(\mathbb{Z} / p \mathbb{Z})^{*}$ satisfy $m_{j} \bar{m}_{j} \equiv$ $1 \bmod p$ for all $j$ and $m_{1}+\ldots+m_{k} \equiv 0 \bmod p$. Then

$$
1+\sum_{j=1}^{k} \frac{\eta^{\bar{m}_{j}}}{1-\eta^{\bar{m}_{j}}}=\sum_{z=1}^{p-1}\left(-1+\sum_{j=1}^{k}\left\langle-\frac{m_{j} z}{p}\right\rangle\right) \eta^{z} .
$$

Proof. For every $j$,

$$
\sum_{z=0}^{p-1}\left\langle\frac{m_{j} z}{p}\right\rangle \eta^{z}=\sum_{z}\left\langle\frac{z}{p}\right\rangle \eta^{\bar{m}_{j} z}
$$

hence

$$
\begin{aligned}
& \left(1-\eta^{\bar{m}_{j}}\right) \sum_{z}\left\langle\frac{m_{j} z}{p}\right\rangle \eta^{z}=\sum_{z}\left\langle\frac{z}{p}\right\rangle\left(\eta^{\bar{m}_{j} z}-\eta^{\bar{m}_{j}(z+1)}\right)= \\
& =\sum_{z=0}^{p-1}\left(\left\langle\frac{z}{p}\right\rangle-\left\langle\frac{z-1}{p}\right\rangle\right) \eta^{\bar{m}_{j} z}=-1+\frac{1}{p}+\sum_{z=1}^{p-1} \frac{1}{p} \eta^{\bar{m}_{j} z}=-1 .
\end{aligned}
$$

Therefore

$$
\frac{\eta^{\bar{m}_{j}}}{1-\eta^{\bar{m}_{j}}}=-1+\frac{1}{1-\eta^{\bar{m}_{j}}}=-1-\sum_{z}\left\langle\frac{m_{j} z}{p}\right\rangle \eta^{z}=
$$




$$
=\sum_{z=1}^{p-1}\left(1-\left\langle\frac{m_{j} z}{p}\right\rangle\right) \eta^{z}=\sum_{z}\left\langle-\frac{m_{j} z}{p}\right\rangle \eta^{z}
$$

and

$$
\begin{aligned}
& 1+\sum_{j=1}^{k} \frac{\eta^{\bar{m}_{j}}}{1-\eta^{\bar{m}_{j}}}=1+\sum_{z}\left(\sum_{j=1}^{k}\left\langle-\frac{m_{j} z}{p}\right\rangle\right) \eta^{z}= \\
& \sum_{z=1}^{p-1}\left(-1+\sum_{j}\left\langle\frac{-m_{j} z}{p}\right\rangle\right) \eta^{z} .
\end{aligned}
$$

The relation to the Chevalley-Weil result becomes more explicit if we observe that $\operatorname{tr} \psi\left(a^{-1}\right)$ is the sum of the eigenvalues of $a^{-1}$, choosing $a^{-1}$-eigendifferentials as basis of $H(X, \Omega)$. Since the roots of unity $\eta^{z}, z \not \equiv 0 \bmod p$, are linearly independent over $\mathbb{Q}$ and since $1+$ $\sum_{z \neq 0 \bmod p} \eta^{z}=0$, we can draw the following conclusion (valid in more general cases, of course).

Corollary 3. Let a be an automorphism of prime order $p$ of the compact Riemann surface $X$, acting in its fixed points with multipliers $\eta^{-\bar{m}_{j}}$. Then, up to an additive term $c$ independent of $z \neq 0$, the coefficient of $\eta^{z}, z \neq 0$, given by Lemma 8 is the dimension

$$
c+\left(-1+\sum_{j}\left\langle\frac{-m_{j} z}{p}\right\rangle\right)
$$

of the eigenspace for the eigenvalue $\eta^{z}$ if we decompose $H^{0}(X, \Omega)$ in eigenspaces for the action of $a^{-1}$. The number $c$ gives the dimension of the subspace of a-invariant differentials (i.e. those lifted from $Z_{p} \backslash X, Z_{p}=\langle a\rangle$ ), hence the genus of $Z_{p} \backslash X$.

Remark 9. If we replace $p$ by $q$ and $\eta$ by $\zeta$ we can apply Lemma 8 also to $\operatorname{tr} \psi\left(a^{m} b^{-y}\right)$ on $X_{n, t, v}$ given by Lemma 7 . For $y \not \equiv 0 \bmod q$ the result is the same as for the representation $\rho$ of $Z_{q}=\langle b\rangle$ on the intermediate covering $Y_{n, t, v}$ (see Remark 5). Corollary 3 applies with $c=0$ and gives the well-known eigenspace decomposition for this curve whose Jacobian has complex multiplication by $\mathbb{Q}(\zeta)$, see $[\mathrm{KR}]$.

If we denote the representation $\psi$ belonging to the surface $X_{n, t, v}$ more precisely by $\psi_{n, t, v}$ we obtain the following 
Theorem 4. The Belyi surfaces $X_{n, t, v}$ and $X_{m, s, w}$ belong to the same Galois orbit if and only if there is a $\sigma \in \mathrm{Gal} \overline{\mathbb{Q}} / \mathbb{Q}$ with the property

$$
\sigma\left(t r \psi_{n, t, v}(g)\right)=\operatorname{tr} \psi_{m, s, w}(\tau g) \text { for all } g \in G \text { and a fixed } \tau \in \text { Aut } G \text {. }
$$

Proof. The Main Observation indicates that Galois conjugate surfaces (see Theorem 3) lead to Galois conjugate traces. On the other hand, if we have Galois conjugated traces for the triples $(n, t, v)$ and $(m, s, w)$, Lemma 3, Lemma 7.2 and Lemma 8 imply the existence of some $k \in$ $(\mathbb{Z} / q \mathbb{Z})^{*}$ with

$$
-1+\left\langle\frac{k n z}{p}\right\rangle+\left\langle\frac{k t z}{p}\right\rangle+\left\langle\frac{k v z}{p}\right\rangle=-1+\left\langle\frac{m z}{p}\right\rangle+\left\langle\frac{s z}{p}\right\rangle+\left\langle\frac{w z}{p}\right\rangle
$$

for all $z \in(\mathbb{Z} / q \mathbb{Z})^{*}$, and by $[\mathrm{KR}]$ this is possible only for equivalent triples. Then Theorem 3 says that the surfaces are Galois conjugate.

The explicit decomposition of the character $t r \psi$ into irreducible characters is of some interest for several questions such as the explicit computation of equations for the canonical model [St1] or more detailed information about the Jacobians [Wo2]. Therefore we include this decomposition for the convenience of the reader but we omit the proof since it can be obtained by standard computations of multiplicities.

Theorem 5. The decomposition of the representation $\psi$ into irreducible components is given by the decomposition of its trace into the irreducible characters of $G$ (see Lemma 1.6) as follows.

1. On $X_{s}$ we have

$$
t r \psi=\sum_{j=1}^{r}\left(-1+\sum_{w \in S}\left\langle-\frac{w v_{j}}{p}\right\rangle\right) \phi_{j} .
$$

2. We consider the representation $\rho$ introduced in Remark 4 as a component of $\psi$ defined on the $(q-1) / 2$-dimensional $\psi(G)$-invariant subspace of $H^{0}\left(X_{n, t, v}, \Omega\right)$ of differentials lifted from $Y_{n, t, v}$. Then we have on $X_{n, t, v}$

$$
\operatorname{tr} \psi=\operatorname{tr} \rho+\frac{q-3}{2} \sum_{j=1}^{r} \phi_{j},
$$




$$
\operatorname{tr} \rho=\sum_{k=1}^{q-1}\left(-1+\left\langle\frac{n k}{q}\right\rangle+\left\langle\frac{t k}{q}\right\rangle+\left\langle\frac{v k}{q}\right\rangle\right) \chi_{k} .
$$

\section{References}

[Be] J. Bertin, Compactification des schémas de Hurwitz. C.R. Acad. Sci. Paris 322, Série I (1996), 1063-1066.

[CW] Cl. Chevalley, A. Weil, ber das Verhalten der Integrale 1. Gattung bei Automorphismen des Funktionenkrpers, Abh. math. Sem. Hamburg 10 (1934), 358-361.

[CG] J-M. Couveignes, L. Granboulan, Dessins from a geometric point of view, pp. 79-113 in L.Schneps (ed.): The Grothendieck Theory of Dessins d'Enfants. London Math. Soc. Lecture Note Series 200, Cambridge UP 1994.

[DE] P. Dèbes, M. Emsalem, On Fields of Moduli of Curves, J. of Algebra 211 (1999), 42-56.

[FK] H.M. Farkas, I. Kra, Riemann Surfaces. Springer GTM 71, 1980.

[GAP] Martin Schönert et al., GAP-Groups, Algorithms and Programming. Lehrstuhl für Mathematik, Rheinisch Westfälische Technische Hochschule, Achen, Germany, sixth edition, 1997.

[JL] G. James, M. Liebeck, Representations and Characters of Groups. Cambridge UP 1993.

[JS] G.A. Jones, D. Singerman, Belyĩ Functions, Hypermaps and Galois Groups, Bull. London Math. Soc. 28 (1996), 561-590.

[JSt] G.A. Jones, M. Streit, Galois Groups, Monodromy Groups and Cartographic Groups, pp. 25-65 in L.Schneps, P.Lochak (ed.): Geometric Galois Actions 2. London Math. Soc. Lecture Note Series 243, Cambridge UP 1997.

[KR] N. Koblitz, D. Rohrlich, Simple factors in the Jacobian of a Fermat curve. Can. J. Math. 30 (1978), 1183-1205.

[K] R.S. Kulkarni, Isolated points in the branch locus of the moduli space of compact Riemann surfaces, Ann. Acad. Sc. Fenn., Ser.A, I. Mathematica 16 (1991), 71-81.

[L] S. Lang, Algebra. Addison-Wesley 1965. 
[Mc] A.M. Macbeath, Action of automorphisms of a compact Riemann surface on the first homology group, Bull. London Math. Soc. 5 (1973), 103-108.

[Po] H. Popp, On a conjecture of H. Rauch on theta constants and Riemann surfaces with many automorphisms, J. Reine Angew. Math. 253 (1972), 66-77.

[Sps] L. Schneps, Dessins d'enfants on the Riemann sphere, pp. 47-77 in L. Schneps (ed.): The Grothendieck Theory of Dessins d'Enfants. London Math. Soc. Lecture Note Series 200, Cambridge UP 1994.

[Si] D. Singerman, Finitely Maximal Fuchsian Groups, J. London Math. Soc. (2) 6 (1972), 29-38.

[SSy] D. Singerman, R.I. Syddall, Belyı̃ Uniformization of Elliptic Curves, Bull. London Math. Soc. 139 (1997), 443-451.

[St1] M. Streit, Homology, Belyĩ Functions and Canonical Curves, manuscripta math. 90 (1996), 489-509.

[St2] M. Streit, Field of Definition and Galois Orbits for the MacbeathHurwitz curves, to appear in Arch. Math.

[Wo1] J. Wolfart, The 'Obvious' Part of Belyǐ's Theorem and Riemann Surfaces with Many Automorphisms, pp. 97-112 in L. Schneps, P. Lochak (ed.): Geometric Galois Actions 1. London Math. Soc. Lecture Note Series 242, Cambridge UP 1997.

[Wo2] J. Wolfart, Triangle groups and Jacobians of CM type, preprint, www.math.uni-frankfurt.de.

Mathematisches Seminar der J.W.Goethe-Universität

Robert-Mayer-Str. 6-10,

D-60054 Frankfurt a.M.

Germany

E-mail address: streit@math.uni-frankfurt.de

E-mail address: wolfart@math.uni-frankfurt.de

Recibido: 26 de Noviembre de 1998

Revisado: 17 de Junio de 1999 